\title{
Intraoperative echocardiographic imaging of coronary arteries and graft anastomoses during coronary artery bypass grafting without cardiopulmonary bypass
}

Yoshihiro Suematsu, MD

Shinichi Takamoto, MD

Toshiya Ohtsuka, MD

From the Department of Cardiothoracic Surgery, University of Tokyo, Tokyo, Japan.

Received for publication March 30, 2001; revisions requested May 17, 2001; revisions received May 29, 2001; accepted for publication June 1, 2001

Address for reprints: Yoshihiro Suematsu, MD, Department of Cardiothoracic Surgery, University of Tokyo, 7-3-1 Hongo Bunkyoku, Tokyo, 113-8655, Japan (E-mail: sue matsu@aurora.dti.ne.jp)

J Thorac Cardiovasc Surg 2001;122:1147-54 Copyright (C) 2001 by The American Association for Thoracic Surgery 0022-5223/2001 $\$ 35.00+0 \quad \mathbf{1 2 / 1 / 1 1 7 6 2 5}$ doi:10.1067/mtc.2001.117625
Background: No accepted approach exists for the intraoperative evaluation of the quality of coronary arteries and the technical adequacy of graft anastomoses during coronary artery bypass grafting without cardiopulmonary bypass.

Methods and Results: We assessed the accuracy of high-frequency epicardial echocardiography and power Doppler imaging in evaluating coronary arteries during coronary artery bypass grafting without cardiopulmonary bypass. To validate measurements of coronary arteries and graft anastomoses by high-frequency epicardial echocardiography and power Doppler imaging, we compared luminal diameters determined by these methods with diameters determined histologically in a study of off-pump coronary artery bypass grafting in 20 dogs. Technical errors were deliberately created in 10 grafts (stenosis group). The results of these animal validation studies showed that the maximum luminal diameters of coronary arteries and graft anastomoses measured by high-frequency epicardial echocardiography (HEE) and power Doppler imaging (PDI) correlated well with the histologic measurements: $\mathrm{HEE}=1.027 \times$ Histologic measurements $+0.005(P<.0001) ;$ PDI $=0.886 \times$ Histologic measurements $+0.0453(P=.0001)$. Similar results were found in the evaluation of the stenosis group: PDI $=0.991 \times$ Histologic measurements $+0.074(P$ $<.0001)$. Subsequently, we demonstrated the clinical applicability of this approach in 12 patients who underwent minimally invasive or off-pump coronary artery bypass grafting. Twenty graft anastomoses were examined intraoperatively by highfrequency epicardial echocardiography and power Doppler imaging, and luminal diameters determined by power Doppler imaging were compared with those determined by postoperative coronary angiography. The results demonstrated that graft anastomosis by power Doppler imaging correlated well with the angiographic measurements: PDI $=1.018 \times$ Angiographic measurements $-0.106(P<.0001)$.

Conclusion: High-frequency epicardial echocardiography can provide meaningful information on the target coronary artery, and power Doppler imaging can accurately measure graft anastomoses and can detect technical errors and inadequacies during coronary artery bypass grafting without cardiopulmonary bypass.

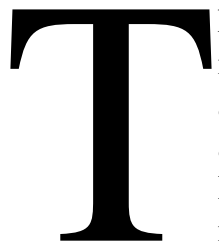

he deleterious effects of cardiopulmonary bypass (CPB) make minimally invasive direct coronary artery bypass grafting (MIDCAB) and off-pump coronary artery bypass grafting (OPCAB) appealing alternatives to the management of coronary artery disease. ${ }^{1-3}$ However, performance of the graft-to-coronary anastomosis is definitely more difficult in the MIDCAB or OPCAB procedures than in conventional coronary artery bypass grafting (CABG) with $\mathrm{CPB},{ }^{4}$ so that intraoperative assessment of the quality of the anastomosis is even more important. Unfortunately, no proven reliable technique for intraoperative assessment of the 

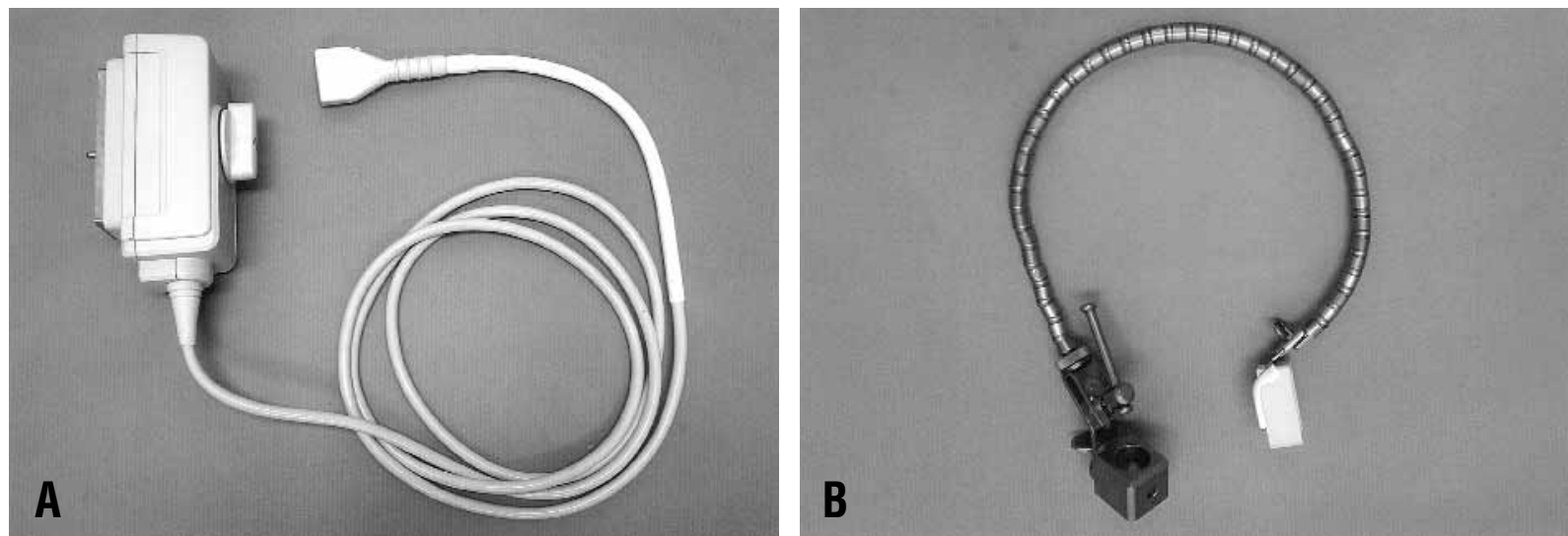

Figure I. A, The 13-MHz echocardiographic probe. This probe is $39 \mathrm{~mm}$ in length and has a $36 \times 10$-mm contact surface. B, The stabilizer makes fixation of the echocardiographic probe possible.

quality of anastomoses exists, except for angiography. Although angiography is universally considered the "gold standard" technique in the assessment of coronary artery disease and graft patency, it can be invasive, costly, time consuming, and not always readily available in the operating room.

High-frequency epicardial echocardiography (HEE) has been used intraoperatively to assess the morphology and geometry of the coronary arteries, ${ }^{5-7}$ and it can also locate coronary arteries that are deeply embedded in epicardial fat or myocardium or that are obscured by epicardial scarring. ${ }^{8}$ Power Doppler imaging (PDI), on the other hand, has been reported to be a feasible method of intraoperative assessment of graft patency. Ishikura and associates ${ }^{9}$ reported an excellent correlation between the diameters of the coronary arteries measured by PDI and quantitative coronary angiography in patients who underwent conventional CABG $(r=$ $0.964, P<.0001)$. However, previous studies were all conducted during conventional CABG. Therefore, the purposes of this study were (1) to validate the accuracy of HEE measurements of native coronary artery in the beating heart in an animal preparation, (2) to validate the accuracy of PDI in assessing graft anastomoses during OPCAB and test the ability of this method in detecting technical errors at graft anastomoses in an animal preparation, and (3) to evaluate patient CABG anastomoses intraoperatively by HEE and PDI. To our knowledge, this is the first study to intraoperatively evaluate the quality of native coronary arteries and graft anastomoses in MIDCAB or OPCAB by echocardiographic imaging.

\section{Materials and Methods \\ Echocardiographic Equipment}

The imaging probe has a scanning frequency of $13 \mathrm{MHz}$. The probe used for animal experiments is approximately $60 \mathrm{~mm}$ in length and has a $52 \times 10$-mm contact surface; the probe used for clinical studies is $39 \mathrm{~mm}$ in length and has a $36 \times 10-\mathrm{mm}$ contact surface, which makes it possible to evaluate posterior circumflex or right coronary artery branches without hemodynamic compromise (Figure 1, $A$ ). The probe also has a depth of field of $2.0 \mathrm{~cm}$ and a 2-point phantom resolution of $0.1 \mathrm{~mm}$, and it can be sterilized by standard ethylene oxide techniques according to the directions for use. The imaging probe is covered with a sterilized pack and sterilized jelly, which allows clear visualization of the anterior wall of the vessels. It is connected to a commercially available scanner for animal studies (LOGIQ 500; GE Yokokawa Systems Ltd, Tokyo, Japan) and for patient studies (SSD-5500; Aloka Co, Ltd, Tokyo, Japan). Real-time images are displayed on a television monitor and recorded on a standard videocassette recorder.

\section{Animal Study}

Twenty mongrel dogs (15-21 kg) were randomly assigned to a control group $(n=10)$ or a stenosis group $(n=10)$. Anesthesia was induced with nembutal (30 mg/kg intravenously) and maintained with $2 \%$ isoflurane. Respiration was maintained on $100 \%$ oxygen with a volume control respirator. The electrocardiogram was continuously monitored throughout the procedure. Arterial blood gases were determined every 30 minutes, and bicarbonate was added as needed to maintain a physiologic $\mathrm{pH}$ between 7.35 and 7.45. Aortic blood pressure was monitored with a $5 \mathrm{~F}$ micromanometer-tipped catheter (Millar Instruments, Inc, Houston, Tex) introduced through the left femoral artery. After median sternotomy, the left internal thoracic artery was dissected from its origin to its bifurcation and wrapped with gauze soaked in papaverine solution $(1 \mathrm{~mL}$ of papaverine diluted in $10 \mathrm{~mL}$ of normal saline solution).

The pericardium was opened and the margins sutured to the edges of the wound. The left anterior descending artery distal to the first diagonal branch was selected for left internal thoracic artery grafting. The anastomotic region was controlled proximally and distally with 3-0 Prolene snares (Ethicon, Inc, Somerville, NJ). Heparin $(1 \mathrm{mg} / \mathrm{kg}$ ) was given intravenously before opening the site of the left anterior descending artery selected. A cardiac stabilizer we modified to permit HEE was used to mechanically stabilize the graft site (Figure 1, $B$ ). This stabilizer consists of two paddles with a malleable segment comparable with that in other commercially available stabilizers, and 
the paddle is covered with short panels, which make fixing of the echocardiographic probe possible. The anastomosis of the left internal thoracic artery to the left anterior descending artery was then constructed "off-pump" by use of 7-0 continuous Prolene sutures. After completion of the anastomosis, intensive stenosis in the stenosis group was created by placing one or two additional blind sutures through the toe of the anastomosis (8-0 Prolene sutures) to reduce the cross-sectional area, as described previously. ${ }^{10}$

\section{Validation and Detection of Technical Errors}

An experienced echocardiographer who was unaware of either the presence or specific type of the technical error scanned the vessels with the imaging probe in the short-axis (cross-sectional) and longitudinal sections, with particular attention to visualization of the native coronary arteries at the maximum diameter. The images were recorded on videotape, and the echocardiographic recordings were measured later with a digitizing tablet. The graft anastomosis was assessed by PDI, and descriptions of surgical technique and images were then compared. After the recordings had been completed, each heart was prepared for histologic examination. The heart was removed, and the vessels were perfused with a barium, formalin, and gelatin mixture at the same mean perfusion pressure that had been recorded during the HEE and PDI evaluations. After the vessels and grafts had been perfusion fixed, the entire heart was placed in $10 \%$ formalin solution and fixed for at least 48 hours. After fixation, the graft-artery anastomosis was removed en bloc and embedded in paraffin. The region was then sectioned and stained with Verhoeff-van Gieson stain for histologic evaluation, as previously described. ${ }^{5}$ Multiple sections were examined to ensure that the maximum diameters of the native coronary arteries and graft anastomosis were identified. The maximum luminal diameters of the anastomoses determined by HEE or PDI and by histologic examination were then compared.

All animals received humane care in compliance with the "Guide for the Care and Use of Laboratory Animals" prepared by the Institute of Laboratory Animal Resources, National Research Council, and published by the National Academy Press, revised 1996, and with the approval of the University of Tokyo Institutional Animal Care and Use Committee.

\section{Patient Study}

The patient study was approved by the Human Subject Review Committee of the University of Tokyo, and written informed consent was obtained according to the institutional guidelines.

Twenty bypass grafts in 12 consecutive patients undergoing an elective MIDCAB (4 patients) or OPCAB operation (8 patients) were studied. Two of the 12 patients underwent triple bypass, 4 had double bypass, and the other 6 received a single bypass graft. The average age of the patients was $67.3 \pm 7.2$ years, and there were 2 women and 10 men. One patient was having a reoperation. The bypass material used was the left internal thoracic artery in 8 grafts, the saphenous vein in 9, the right gastroepiploic artery in 1, and the radial artery in 2. Target vessels were the left anterior descending artery in 11 , the right coronary artery in 5 , the left circumflex branch in 2, and the diagonal branch in 2 patients.

Standard anesthetic management and exposure of the heart through a median sternotomy or a small left thoracotomy were used in all patients. Radial artery pressure, left atrial pressure, and

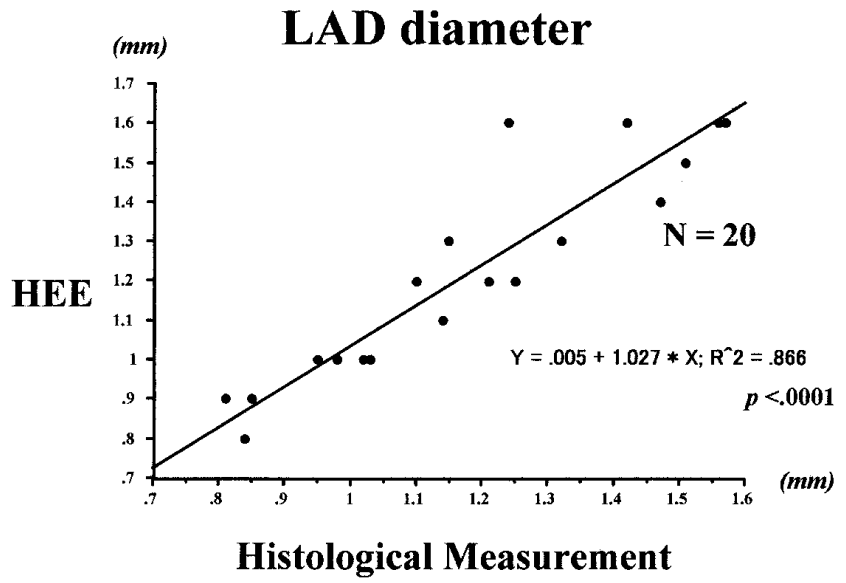

Figure 2. Comparison between the maximum luminal diameter of the left anterior descending (LAD) artery measured by high-frequency epicardial echocardiography (HEE) and that measured histologically in the animal study.

the electrocardiogram were continuously monitored. The left internal thoracic artery was dissected from its origin to its bifurcation and wrapped with gauze soaked in papaverine solution in the usual fashion. If needed, saphenous veins, right gastroepiploic arteries, or radial arteries were harvested. During OPCAB operations, 3 deep pericardial traction stitches were placed near the left upper and lower pulmonary veins and to the left of the inferior vena cava, thereby elevating the apex of the heart. With perfectly placed stitches and aggressive traction, the apex of the heart should be elevated to about $90^{\circ}$. To further assist in providing good exposure of the target arteries on the lateral and inferior aspect of the hearts, patients were placed in a gentle right decubitus Trendelenburg position. Our stabilizer was used to stabilize the target arteries. HEE was first applied to identification of native coronary arteries with walls suitable for anastomosis. If calcification or atherosclerotic plaques were present, the site of anastomosis was changed. All distal anastomoses were constructed with 7-0 or 8-0 Prolene suture by a continuous technique. The quality of the anastomoses was assessed at the time of the operation in all cases in which PDI was used.

\section{Statistical Analysis}

Statistical analysis was performed with the StatView (version 5) software package (SAS Institute, Inc, Cary, NC). Linear regression analysis was used to correlate paired data sets. Differences were considered significant when confidence limits exceeded $95 \%(P<.05)$.

\section{Results \\ Animal Study}

In the animal studies, maximum luminal diameter of the left anterior descending artery measured by HEE was significantly correlated with that measured histologically (HEE measurements $=1.027 \times$ Histologic measurements +0.005 ; $P<.0001, \mathrm{n}=20$ ) (Figure 2). 


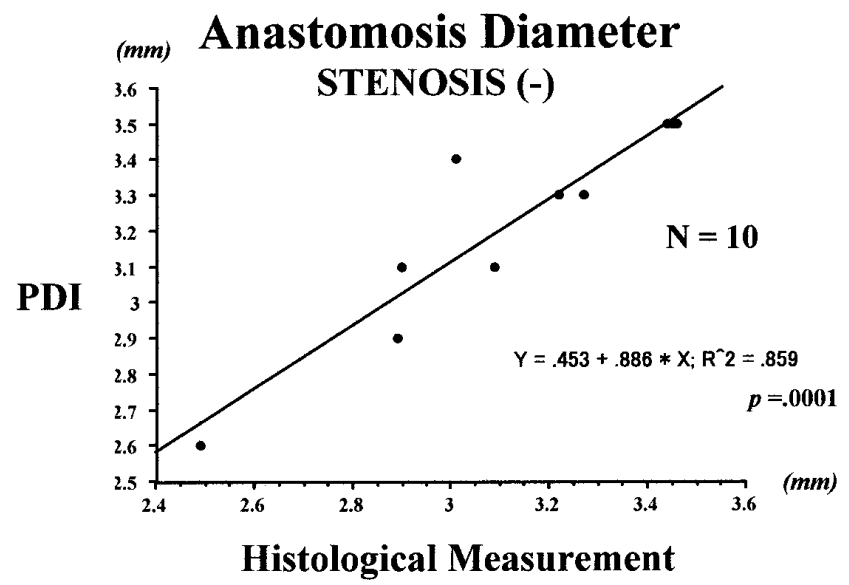

Figure 3. Comparison between the maximum luminal diameter of the left internal thoracic artery-left anterior descending artery anastomosis measured by power Doppler imaging (PDI) and histologically in the animal study.

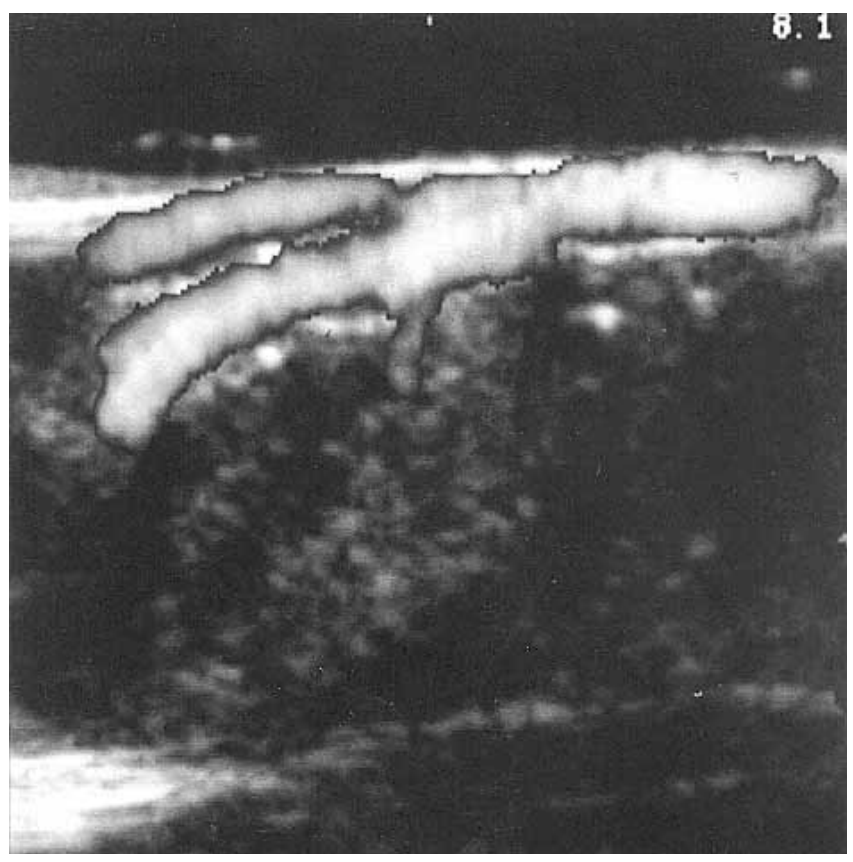

Figure 4. Power Doppler image showing a longitudinal section of the left internal thoracic artery-left anterior descending artery anastomosis in the stenosis group.

All 10 anastomoses in which deliberate technical errors had been made were detected by the independent observer. The morphologic features of the technical error agreed well with the error predicted and recorded by the surgeon. Figure 3 shows representative PDI images in the stenosis group. Comparisons of the maximum luminal diameter measurements of the anastomosis by PDI with histologic measure-

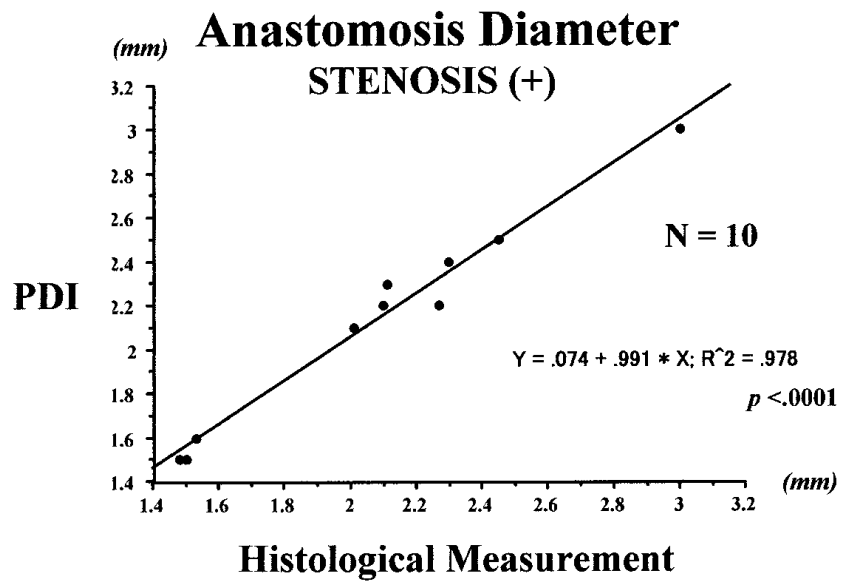

Figure 5. Comparison between the maximum luminal diameter of the left internal thoracic artery-left anterior descending artery anastomosis measured by power Doppler imaging (PDI) and histologically in the stenosis group in the animal study.

ments in the control group and stenosis group are shown in Figures 4 and 5. Correlations between the 2 methods were excellent (control group: PDI measurements $=0.886 \times$ Histologic measurements $+0.0453 ; P=.0001, \mathrm{n}=10$; stenosis group: PDI measurements $=0.991 \times$ Histologic measurements $+0.074 ; P<.0001, \mathrm{n}=10)$.

\section{Patient Study}

Echocardiographic images of high quality were easily obtained in patient studies by the approach described above. In 3 patients, the site of anastomosis was changed because of dense calcification. Deeply embedded coronary arteries could be precisely located within about 1 minute, without extensive and time-consuming dissection. Figure 6 shows sample intraoperative images of native coronary arteries obtained by HEE. Atherosclerotic plaques and calcifications were detected in the native coronary arteries. Figure 7 shows examples of graft anastomoses obtained by PDI. No abnormalities were noted in the anastomoses. In addition, the septal perforator branches were easily visualized by PDI.

The maximum luminal diameter measurements of graft anastomosis determined by intraoperative PDI and postoperative coronary angiography are compared in Figure 8, and an excellent correlation was found between the results obtained by these 2 methods (PDI measurements $=1.018 \times$ Angiographic measurements $-0.106 ; P<.0001, \mathrm{n}=20$ ).

No complications related to epicardial imaging occurred, such as injury to coronary vessels, compromise, infection, or electrical or mechanical hazards.

\section{Discussion}

The use of MIDCAB and OPCAB has recently been increasing because of increasing numbers of high-risk patients, their 

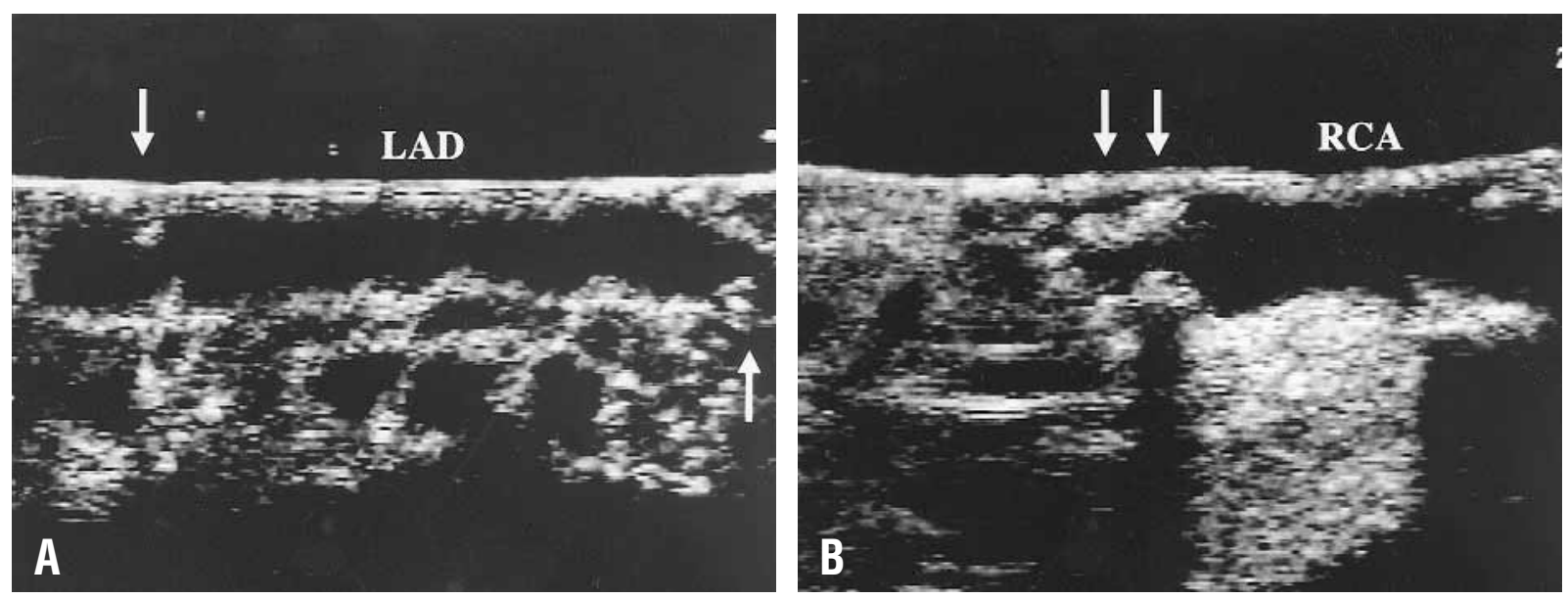

Figure 6. A, High-frequency epicardial echocardiographic image obtained in the patient study showing a longitudinal section of left anterior descending artery (LAD). Arrows are pointing to atherosclerotic plaque. B, Highfrequency epicardial echocardiographic image obtained in the patient study showing a longitudinal section of right coronary artery (RCA). Arrows are pointing to atherosclerotic plaque.
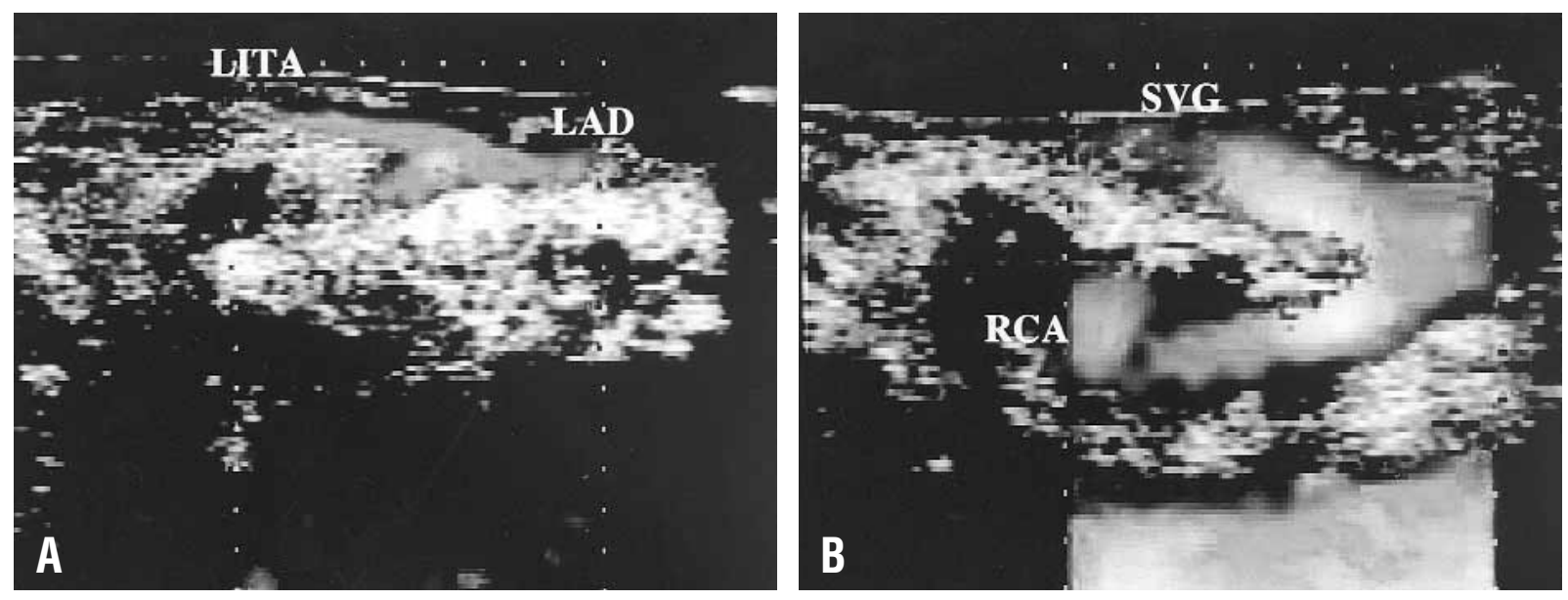

Figure 7. A, Power Doppler image of the left internal thoracic artery (LITA) anastomosis to the left anterior descending artery (LAD). B, Power Doppler image of a saphenous vein graft (SVG) anastomosed to the right coronary artery (RCA).

quality equivalence to conventional $\mathrm{CABG}$, and their cost effectiveness. ${ }^{1-3}$ Although the introduction of cardiac stabilizers has significantly facilitated these procedures, the probability of a technical error is greater than with conventional CABG with the aid of the CPB machine and cardioplegic arrest. $^{4,11}$ Waller and Roberts ${ }^{12}$ suggested that the inadvertent placement of grafts proximal to stenotic plaques may play a role in graft occlusion. It is therefore important for surgeons to find a reliable method of assessing anastomoses so that MIDCAB or OPCAB will become more universally accepted.

Coronary angiography is generally considered the "gold standard" to which all other methods should be compared.
However, it is invasive, costly, time consuming, and not always readily available in the operating room.

Thermal coronary artery imaging with an infrared camera was introduced by Falk and colleagues ${ }^{13}$ for conventional CABG, but the quality of the available technology only allows detection of a grossly stenosed anastomosis. Not only does it not allow visualization of the posterior circumflex or right coronary artery branches, but of intramyocardial arteries and arteries surrounded by fat as well. Thermal coronary imaging is only suitable when temperature gradients exist.

A variety of flow measurement techniques have frequently been used for intraoperative assessment of the qual- 


\section{Anastomosis Diameter}

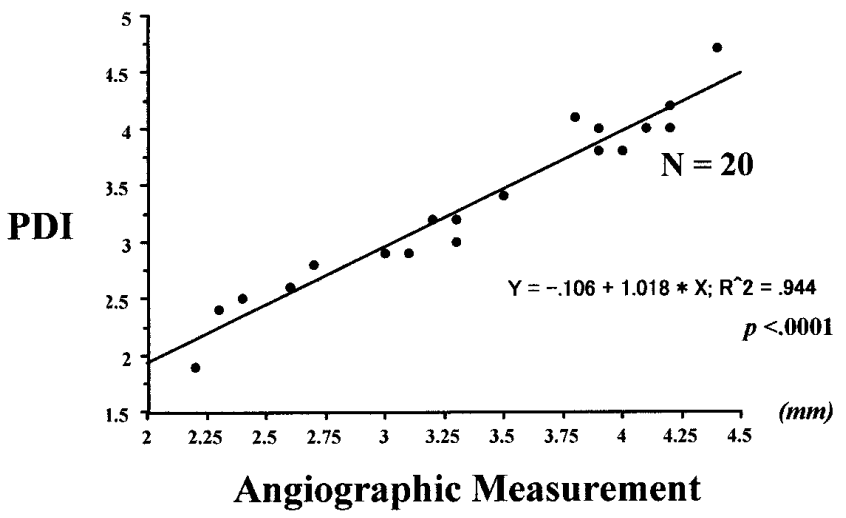

Figure 8. Comparison between the maximum luminal diameter of the graft anastomosis measured by intraoperative power Doppler imaging (PDI) and by postoperative angiography in the patient study.

ity of anastomoses. ${ }^{14,15}$ Because of the peculiar physiology of the coronary circulation, flow through the coronary grafts occurs during diastole with a short systolic peak. Flow probes provide continuous flow tracings and mean flows through the graft with a high degree of accuracy. Despite being able to measure flow accurately, in their experimental study, Jaber and associates ${ }^{10}$ documented that graft flow was not reliably well correlated with anastomotic quality. They also concluded that lesser degrees of stenosis at risk for failure may go undetected, and patent grafts may inadvertently be redone.

Echocardiography has been used in an attempt to visualize coronary arteries in closed-chest patients, and the usefulness of HEE in evaluating the luminal diameter, area, and wall thicknesses of native coronary arteries has been demonstrated.$^{5-7}$ In addition to allowing assessment of morphologic features, intraoperative HEE can locate coronary arteries that are deeply embedded in epicardial fat or myocardium or that are obscured by epicardial scarring, ${ }^{8}$ and the nature and precise location of atherosclerotic plaques can be easily determined. Hiratzka and coworkers ${ }^{16}$ intraoperatively evaluated patients' bypass graft anastomoses by HEE. They concluded that HEE could accurately measure bypass graft anastomoses and had the potential to intraoperatively detect technical errors and inadequacies.

Ishikura and coworkers ${ }^{9}$ demonstrated the efficiency of PDI in visualizing coronary arteries in clinical settings. Power Doppler ultrasonography, which is based on the total integrated power of the Doppler spectrum, has several advantages over conventional color Doppler echocardiography: it is more sensitive to visualization of smaller vessels, is angle-independent, and does not produce signal alias-
TABLE 1. Echocardiographic method for CABG

\begin{tabular}{lcc}
\hline & HEE & PDI \\
\hline $\begin{array}{l}\text { Location } \\
\quad \text { Coronary artery buried deeply in fat } \\
\quad \text { or myocardium }\end{array}$ & & \\
$\quad$ Coronary artery under epicardial scar & $\bigcirc$ & $\triangle$ \\
$\quad$ Perforator & $\triangle$ & $\bigcirc$ \\
Morphology & & \\
$\quad$ Atherosclerotic plaque & $\bigcirc$ & $\mathrm{X}$ \\
$\quad$ Wall thickness & $\bigcirc$ & $\mathrm{X}$ \\
$\quad$ Luminal diameter & $\bigcirc$ & $\triangle$ \\
$\quad$ Calcification & $\mathrm{X}$ & $\mathrm{X}$ \\
Visualization & & \\
$\quad$ Graft anastomosis & & \\
$\quad$ Myocardial perfusion & $\triangle$ & $\bigcirc$ \\
\hline
\end{tabular}

$H E E$, High-frequency epicardial echocardiography; PDI, power Doppler imaging; $\bigcirc$, good; $\triangle$, fair; $X$, poor.

ing. ${ }^{17,18}$ Arruda and colleagues ${ }^{19}$ applied PDI to visualization of myocardial perfusion after anastomosis of the left internal thoracic artery to the left anterior descending coronary artery. They concluded that visualization of bypass grafts was feasible in all patients and that PDI allowed rapid and simple intraoperative assessment of graft patency. Precise location of septal perforator branches is also possible. In the present study, PDI was very useful in choosing the site to snare native coronary arteries to prevent back flow from those branches during anastomosis.

We selected HEE to evaluate native coronary arteries and PDI for graft anastomoses in our study. In a human autopsy study, McPherson and colleagues ${ }^{5}$ demonstrated that coronary artery wall thickness measurements correlated well with HEE measurements $(r=0.86$; HEE measurements $=0.65 \times$ Histologic measurements +0.24$)$. They concluded that HEE is an accurate and reproducible method of measuring coronary luminal and wall geometry and a potentially useful tool for in vivo coronary artery evaluation of patients. On the other hand, PDI displays information on vessel perfusion, but not on coronary luminal and wall geometry. Conversely, vessel perfusion is more important for graft anastomosis than information on coronary luminal and wall geometry. Besides, switching from HEE to PDI is easy because both methods can be performed with the same probe and imaging can be switched in a few seconds. We therefore recommend combined use of these methods (Table 1).

As mentioned earlier, previous studies were all conducted during cardiac arrest with cardioplegia or minimized cardiac motion during $\mathrm{CPB}$, and cardiac motion has been a major problem during intraoperative studies in MIDCAB or OPCAB. It was thought that applying the imaging probe was 
difficult and that reproducible results would not be obtained under such conditions. In this study, we developed a suitable stabilizer that effectively immobilizes the local heart surface by light compression and permits HEE. It was possible to safely immobilize the coronary artery during MIDCAB or $\mathrm{OPCAB}$ and to evaluate both the quality of the wall of the native coronary arteries and the graft anastomoses.

Some studies have documented that the large size of the hand-held imaging probe currently used does not permit imaging of coronary arteries or bypass grafts to posterior circumflex or right coronary artery branches without hemodynamic compromise. ${ }^{8,20}$ Since the size of the probe we used in the animal study was relatively large and did not allow placement over those arteries, we recently developed a new smaller probe and stabilizer that can be placed over these arteries except on the deep left circumflex branch. The probe costs about $\$ 12,000$. In addition, this method is simple to use and highly accurate. Although we did not evaluate the technical variance in the present study, a previous study, in which the intraobserver and interobserver variability were also examined, showed excellent reproducibility for the measurement of HEE. ${ }^{21}$ Therefore, we believe that this technique can be applied widely.

The time required to obtain and analyze an intraoperative image is approximately 1 minute per anastomosis. Clinically important findings such as the presence of a major graft obstruction can be extracted from videotapes in the stopframe mode almost immediately. The time required for echocardiographic examinations when revision of the anastomosis is needed might be considered excessive. The potential benefit of correcting an otherwise unsatisfactory anastomosis, however, should outweigh the risk of the additional time.

Both HEE and PDI may have limitations in visualizing calcified structures $^{21}$ and soft plaque. ${ }^{22}$ However, vessel walls with calcifications or plaques are not suitable for anastomosis, and the anastomosis site was usually created on the arteries without calcification or plaques. It therefore appears that the limitations in visualizing calcified structures is not a major problem for application of $\mathrm{HEE}$ and PDI during MIDCAB or OPCAB.

\section{Conclusions}

In this study, we have shown that HEE can accurately measure the dimensions of native coronary arteries and that technical abnormalities of graft anastomoses can be detected with PDI in the presence of a beating heart. In addition, we have demonstrated that patient graft anastomoses can be quickly evaluated intraoperatively with HEE and PDI during MIDCAB or OPCAB.

We acknowledge the echocardiographic technical support of Ryota Amagai, Tatsuya Maki, and Atsushi Koyama in the preparation of this manuscript.

\section{References}

1. Mehran R, Dangas G, Stamou SC, Pfister AJ, Dullum MK, Leon MB, et al. One-year clinical outcome after minimally invasive direct coronary artery bypass. Circulation. 2000;102:2799-802.

2. Bowles BJ, Lee JD, Dang CR, Taoka SN, Johnson EW, Lan EM, et al. Coronary artery bypass performed without the use of cardiopulmonary bypass is associated with reduced cerebral microemboli and improved clinical results. Chest. 2001;119:25-30.

3. Pfister AJ, Zaki MS, Garcia JM, Mispireta LA, Corso PJ, Qazi AG, et al. Coronary artery bypass without cardiopulmonary bypass. Ann Thorac Surg. 1992;54:1085-92.

4. Gundry SR, Romano MA, Shattuck OH, Razzouk AJ, Bailey LL. Seven-year follow-up of coronary artery bypasses performed with and without cardiopulmonary bypass. J Thorac Cardiovasc Surg. 1998; 115:1273-7.

5. McPherson DD, Armstrong M, Rose E, Kieso RA, Megan M, Hunt M, et al. High frequency epicardial echocardiography for coronary artery evaluation: in vitro and in vivo validation of arterial lumen and wall thickness measurements. J Am Coll Cardiol. 1986;8:600-6.

6. McPherson DD, Johnson MR, Hunt M, Hiratzka L, Lamberth W, Brandt P, et al. Coronary arterial evaluation: comparison of quantitative coronary angiography to high frequency echocardiography. Circulation. 1985;72(Suppl):III-59.

7. Sahn DJ, Barratt-Boyes BG, Graham K, Kerr A, Roche A, Hill D, et al. Ultrasonic imaging of the coronary arteries in open-chest humans: evaluation of coronary atherosclerotic lesions during cardiac surgery. Circulation. 1982;66:1034-44.

8. Hiratzka LF, McPherson DD, Brandt B 3rd, Lamberth WC Jr, Marcus ML, Kerber RE. Intraoperative high-frequency epicardial echocardiography in coronary revascularization: locating deeply embedded coronary arteries. Ann Thorac Surg. 1986;42:S9-11.

9. Ishikura F, Matsuwaka R, Sakakibara T, Sakata Y, Hirayama A, Kodama K. Clinical application of power Doppler imaging to visualize coronary arteries in human beings. J Am Soc Echocardiogr. 1998; 11:219-27.

10. Jaber SF, Koenig SC, BhaskerRao B, VanHimbergen DJ, Cerrito PB, Ewert DJ, et al. Role of graft flow measurement technique in anastomotic quality assessment in minimally invasive CABG. Ann Thorac Surg. 1998;66:1087-92.

11. Jansen EW, Borst C, Lahpor JR, Grundeman PF, Eefting FD, Nierich A, et al. Coronary artery bypass grafting without cardiopulmonary bypass using the Octopus method: results in the first one hundred patients. J Thorac Cardiovasc Surg. 1998;116:60-7.

12. Waller BF, Roberts WC. Severe coronary narrowing at the site of or distal to the bypass graft anastomosis is the most important cause of early and late graft closure: analysis of 102 necropsy patients (108 arteries and 185 grafts) having an isolated aorto-coronary bypass operation. J Am Coll Cardiol. 1983;2:719-28.

13. Falk V, Walther T, Philippi A, Autschbach R, Krieger H, Dalichau H, et al. Thermal coronary angiography for intraoperative patency control of arterial and saphenous vein coronary artery bypass grafts: results in 370 patients. J Card Surg. 1995;10:147-60.

14. Lundell A, Bergqvist D, Mattsson E, Nilsson B. Volume blood flow measurements with a transit time flowmeter: an in vivo and in vitro variability and validation study. Clin Physiol. 1993;13:547-57.

15. Canver CC, Dame NA. Ultrasonic assessment of internal thoracic artery graft flow in the revascularized heart. Ann Thorac Surg. 1994; 58:135-8.

16. Hiratzka LF, McPherson DD, Brandt B, Lamberth WC, Sima S, Marcus ML, et al. The role of intraoperative high-frequency epicardial echocardiography during coronary artery revascularization. Circulation. 1987;76(Suppl):V-33-8.

17. MacSweeney JE, Cosgrove DO, Arenson J. Colour Doppler energy (power) mode ultrasound. Clin Radiol. 1996;51:387-90.

18. Chen JF, Fowlkes JB, Carson PL, Rubin JM, Adler RS. Autocorrelation of integrated power Doppler signals and its application. Ultrasound Med Biol. 1996;22:1053-7.

19. Arruda AM, Dearani JA, Click RL, Ishikura F, Seward JB. Intraoperative application of power Doppler imaging: visualization of myocardial perfusion after anastomosis of left internal thoracic artery 
to left anterior descending coronary artery. J Am Soc Echocardiogr. 1999;12:650-4.

20. Sahn DJ, Copeland JG, Temkin LP, Wirt DP, Mammana R, Glenn W. Anatomic-ultrasound correlations for intraoperative open chest imaging of coronary artery atherosclerotic lesions in human beings. $J \mathrm{Am}$ Coll Cardiol. 1984;3:1169-77.

21. McPherson DD, Sima SJ, Hiratzka LF, Thorpe L, Armstrong ML,
Marcus ML, et al. Coronary arterial remodeling studied by highfrequency epicardial echocardiography: an early compensatory mechanism in patients with obstructive coronary atherosclerosis. J Am Coll Cardiol. 1991;17:79-86.

22. Barnes RW, Bone GE, Reinertson J, Slaymaker EE, Hokanson DE, Strandness DE. Noninvasive ultrasonic carotid angiography: prospective validation by contrast arteriography. Surgery. 1976;80:328-35.

\section{ON THE MOVE?}

Send us your new address at least six weeks ahead

Don't miss a single issue of the journal! To ensure prompt service when you change your address, please photocopy and complete the form below.

Please send your change of address notification at least six weeks before your move to ensure continued service. We regret we cannot guarantee replacement of issues missed due to late notification.

JOURNAL TITLE:

Fill in the title of the journal here.

\section{OLD ADDRESS:}

Affix the address label from a recent issue of the journal here.

\section{NEW ADDRESS:}

Clearly print your new address here.

Name

Address

City/State/ZIP
COPY AND MAIL THIS FORM TO:

Mosby

Subscription Customer Service

6277 Sea Harbor Dr

Orlando, FL 32887
OR FAX TO:

407-363-9661

N/ Mosby
OR PHONE: 800-654-2452

Outside the U.S., call

407-345-4000 